\title{
Odorant Response Properties of Convergent Olfactory Receptor Neurons
}

\author{
Thomas C. Bozza and John S. Kauer \\ Department of Neuroscience, Tufts University School of Medicine, Boston, Massachusetts 02111
}

Information about odorant stimuli is thought to be represented in spatial and temporal patterns of activity across neurons in the olfactory epithelium and the olfactory bulb (OB). Previous studies suggest that olfactory receptor neurons (ORNs) distributed in the nasal cavity project to localized regions in the glomerular layer of the OB. However, the functional significance of this convergence is not yet known, and in no studies have the odorant response properties of individual ORNs projecting to defined $O B$ regions been measured directly. We have retrogradely labeled mouse ORNs connecting to different glomeruli in the dorsal $O B$ and tested single cells for responses to odorants using fura-2 calcium imaging. ORNs that project to clusters of dorsomedial (DM) glomeruli exhibit different odorant response profiles from those that project to dorsolateral (DL) glomeruli. DL-projecting ORNs showed responses to compounds with widely different structures, including carvone, eugenol, cinnamaldehyde, and acetophenone. In contrast, DMprojecting neurons exhibited responses to a more structurally restricted set of compounds and responded preferentially to organic acids. These data demonstrate that ORN afferents segregate by odorant responsiveness and that the homogeneity of ORN and glomerular input varies with different $O B$ regions. The data also demonstrate that a subpopulation of ORNs projecting to DM glomeruli is functionally similar.

Key words: olfactory receptor neurons; olfactory bulb; calcium; imaging; retrograde tracing; glomeruli; mouse; olfactory coding
Odorant stimuli interact with a vast array of differentially responsive olfactory receptor neurons (ORNs) in the nasal cavity. Single-unit (Duchamp et al., 1974; Getchell, 1974; Revial et al., 1978, 1982), field potential (MacKay-Sim et al., 1982; MackaySim and Kesteven, 1994; Scott et al., 1996, 1997), and opticalimaging (Youngentob et al., 1995) studies in amphibians and rodents have demonstrated that individual odorants differentially activate broad regions of the olfactory epithelium (OE) and that ORNs are "broadly tuned," responding to many, often structurally dissimilar, molecules. In addition, ORNs that are near neighbors in the OE respond differently to odorants (Sato et al., 1994). As a result, it is difficult to predict the response properties of ORNs based on their epithelial locations.

ORNs send single unbranched axons back to the olfactory bulb (OB), where they synapse on dendrites of mitral and tufted cells in spherical regions of neuropil called glomeruli. In mice there are $\sim 1800$ glomeruli that cover the surface of the OB (Royet et al., 1988; Pomeroy et al., 1990). The connections between the OE and the OB lack a strict point-to-point topography; ORNs in broad regions of the $\mathrm{OE}$ converge onto localized regions of the $\mathrm{OB}$ (Kauer, 1980; Astic and Saucier, 1986; Astic et al., 1987; Stewart and Pedersen, 1987; Schoenfeld et al., 1994). Recent molecular data in rodents have shown that spatially distributed ORNs that

\footnotetext{
Received Jan. 12, 1998; revised March 16, 1998; accepted March 25, 1998.

This work was supported by a grant from National Institutes of Health, National Institute on Deafness and Other Communication Disorders. We thank Diego Restrepo for advice and assistance with calcium imaging, A. LaMantia for advice concerning glomerular imaging, and William Rand for help with statistical analyses. We thank Dale Hunter, Joel White, Kathleen Dorries, and Tarik Alkasab for valuable discussion and assistance in preparing this paper.

Correspondence should be addressed to Dr. John S. Kauer, Department of Neuroscience, Tufts University School of Medicine, 136 Harrison Avenue, Boston, MA 02111.

Dr. Bozza's present address: The Rockefeller University, 1230 York Avenue, New York, NY 10021.

Copyright (C) 1998 Society for Neuroscience $\quad 0270-6474 / 98 / 184560-10 \$ 05.00 / 0$
}

express the same putative odorant receptor (OR) gene send convergent projections to pairs of glomeruli in a remarkably precise and reproducible manner (Ressler et al., 1994; Vassar et al., 1994; Mombaerts et al., 1996). This suggests that ORN afferents can segregate by odorant responsiveness and that local regions of the OB may receive inputs from ORNs with similar response properties (Kauer, 1980, 1987; Shepherd, 1994; Mori, 1995). This idea is supported by physiological data suggesting that individual odorants are represented in part by the spatiotemporal activation of ensembles of glomeruli (Stewart et al., 1979; Jourdan et al., 1980; Lancet et al., 1982; Guthrie et al., 1993; Cinelli et al., 1995; Friedrich and Korsching, 1997; Joerges et al., 1997). However, there are few data relating the expression of particular OR sequences with functional properties of ORNs (Zhao et al., 1998), and there is presently no information relating the differential expression of OR sequences with the spatial representation of odorant stimuli. Furthermore, there have been no studies in rodents that directly measure the odorant response properties of ORNs that send convergent projections to identified regions of the OB (Friedrich and Korsching, 1997; Joerges et al., 1997).

In the present paper, we have studied the relationship between odorant response properties of ORNs and their projection sites onto local OB regions. Through a combination of retrograde tracing and calcium imaging, we have recorded from single ORNs that project to defined, localized regions of the dorsal OB. The data provide the first direct characterization of the functional topography of projections between the $\mathrm{OE}$ and $\mathrm{OB}$ in mammals assayed at the level of individual ORNs.

\section{MATERIALS AND METHODS}

Animals. All experiments were performed using postnatal day 12-15 (P12-P15) CF-1 mice (Charles River, Wilmington, MA). Young animals were used to facilitate the surgical manipulations required for glomerular imaging and retrograde tracing. CF-1 mice were chosen because vital 
imaging of glomeruli has been performed in this strain (LaMantia and Purves, 1989; LaMantia et al., 1992), and the postnatal development of the OE and OB has been well studied (Pomeroy et al., 1990).

Retrograde tracing. Mice were anesthetized with ketamine $(90 \mathrm{mg} / \mathrm{kg})$ and xylazine $(18 \mathrm{mg} / \mathrm{kg})$, and the dorsal surface of the left OB was exposed with the dura intact by cutting a $\sim 1.5 \times 1.8 \mathrm{~mm}$ window in the cranium. The windows were positioned with respect to the midline suture between the frontal bones and the posterior end of the $\mathrm{OB}$ and typically exposed $70 \%$ of the dorsal OB. Glomeruli were visualized using methods modified from those of LaMantia et al. (1992). The OBs were stained for 15-25 min with a $2 \mathrm{mg} / \mathrm{ml}$ solution of the fluorescent dye RH414 (Molecular Probes, Eugene, OR). Staining was achieved without pretreatment with hypertonic saline (LaMantia et al., 1992) by sweeping the dura with a cotton swab before applying the dye. Excess dye was removed, and the animals were placed in a head holder mounted to the modified stage of an upright fluorescence microscope. RH414-stained glomeruli and microspheres used for injections were viewed simultaneously with a long-pass fluorescein filter set (excitation, $490 \mathrm{~nm}$; dichroic, $500 \mathrm{~nm}$; and emission, $>515 \mathrm{~nm}$ ) and a PlanApo $4 \times$ objective.

Small injections of green latex microspheres (Katz and Iarovici, 1990) (LumaFluor, Naples, FL) were made into visualized groups of glomeruli in the dorsomedial (DM) and dorsolateral (DL) OB using an injection micropipette (tip size, $\sim 5 \mu \mathrm{m}$ ) connected to a $1 \mathrm{ml}$ glass syringe via Teflon tubing. The entire assembly was filled with Fluorinert FC-77 (Sigma, St. Louis, MO) allowing small volumes of tracer to be sucked up and expelled from the injection micropipette. Placement of the injections was made with reference to the medial and rostral edges of the craniotomy. Although glomerular landmarks were not used to position injections, staining of the glomeruli greatly aided our ability to inject the glomerular layer under fluorescence illumination and permitted injection size to be determined in relation to glomerular size during the injection procedure. Video images of the OBs were acquired before and immediately after injection and before cell dissociation (or histological analysis) to quantify the sizes of the injections and to monitor diffusion of the tracer from the injection site. Based on these images, the mean area of the labeled dorsal glomeruli was $6681.8 \pm 198.6 \mu \mathrm{m}^{2}$ (mean $\pm \mathrm{SEM} ; n=$ 208); the injections encompassed $\sim 21$ glomeruli for the DM site $(0.14 \pm$ $0.03 \mathrm{~mm}^{2}$, mean $\left.\pm \mathrm{SD}\right)$ and $\sim 32$ glomeruli $\left(0.22 \pm 0.08 \mathrm{~mm}^{2}\right)$ for the DL site. Lateral tracer diffusion was minimal during the retrograde tracing period.

In addition, the exact locations of all the injections included in the analyses were determined from video images of the entire exposed OBs (for example, see Fig. 2E,F). Injections outside the DM and DL regions were not included in the analyses. The precise location of the injection site centers with respect to the medial and rostral edges of the OB were measured as fractions of total bulbar width and length after being scaled to a "standard" bulb of average dimensions. The standardized locations of the injection site centers for animals included in the physiological analyses as measured from the medial edge and rostral tip of the OB were $\mathrm{DM}=0.39 \pm 0.09 \times 1.21 \pm 0.09 \mathrm{~mm}$; and $\mathrm{DL}=1.36 \pm 0.11 \times 1.25 \pm$ $0.14 \mathrm{~mm}$ (mean $\pm \mathrm{SD}$ ). Retrograde tracing with RH414 and microspheres was evident in the OE by $24 \mathrm{hr}$. Tracing periods were $1-3 \mathrm{~d}$ for calcium imaging and 3-6 d for histology and whole mounts.

Histology. Labeling was viewed in unfixed tissue, because RH414 fluorescence is abolished by short periods of paraformaldehyde fixation. For cryosections, the intact septum and turbinates were removed and washed in PBS and cryoprotected in PBS and 30\% sucrose overnight. The tissue was then embedded and frozen in O.C.T. and cut into $20 \mu \mathrm{m}$ sections in a freezing microtome. For whole mounts, the septum and turbinates were removed and placed on depression slides in Ringer's solution (below). Whole mounts and sections were viewed and photographed using standard rhodamine and fluorescein filter sets. All images were digitized and assembled for display using Adobe Photoshop 4.0 (Adobe Systems) using the minimum digital image processing necessary to accurately reproduce the data.

Cell dissociation and calcium imaging. Procedures were modified from those of Restrepo et al. (1993). Animals were killed, and the OE was dissected and minced at $4{ }^{\circ} \mathrm{C}$ in low-Ca ${ }^{2+}$ Ringer's solution (in mM: 140 $\mathrm{NaCl}, 5 \mathrm{KCl}, 10 \mathrm{HEPES}, 1$ EDTA, 10 glucose, and 1 sodium pyruvate, $\mathrm{pH}$ 7.2) supplemented with $1 \mathrm{~mm}$ cysteine. The tissue was incubated in 1 $\mathrm{U} / \mathrm{ml}$ papain (Sigma) for $4 \mathrm{~min}$ at room temperature, and the reaction was terminated with $1 \mathrm{ml}$ of Ringer's solution (in mM: $140 \mathrm{NaCl}, 5 \mathrm{KCl}$, $1 \mathrm{CaCl}_{2}, 1 \mathrm{MgCl}_{2}, 10$ HEPES, 10 glucose, and 1 sodium pyruvate, $\mathrm{pH}$
7.2) supplemented with $0.1 \mathrm{mg} / \mathrm{ml} \mathrm{BSA,} 200 \mu \mathrm{g} / \mathrm{ml}$ leupeptin (Sigma), and $0.025 \mathrm{mg} / \mathrm{ml}$ DNase I (Sigma). Cells were washed, resuspended in Ringer's solution with $8 \mu \mathrm{M}$ fura-2 AM and $80 \mu \mathrm{g} / \mathrm{ml}$ Pluronic F-127 (Molecular Probes), gently triturated through a flame-polished Pasteur pipette, plated on glass coverslips (coated with Con A type V, $2 \mathrm{mg} / \mathrm{ml}$; and poly-L-lysine, $1 \mathrm{mg} / \mathrm{ml}$ ), and loaded for $45 \mathrm{~min}-1 \mathrm{hr}$ at room temperature. The coverslips formed the bottom of a Teflon recording chamber (Biophysica, Sparks, MD). Dissociated preparations were constantly perfused with normal Ringer's solution $(\sim 3 \mathrm{ml} / \mathrm{min})$ except during stimulus presentations.

Stimuli were bath-applied for 4-10 sec. Complete washout of the chamber required 1-2 min. The depolarizing stimulus was a high- $\mathrm{K}^{+}$ Ringer's solution (in mM: $40 \mathrm{NaCl}, 100 \mathrm{KCl}, 1 \mathrm{CaCl}_{2}, 1 \mathrm{MgCl}_{2}, 10$ HEPES, 10 glucose, and 1 sodium pyruvate, $\mathrm{pH}$ 7.2). The odorant stimuli were a set of six mixtures (mixes A-F) of 32 different molecules (Fig. 1). Mixes A-D were designed so that each mixture included molecules with various chemical structures and odor qualities. Mix B was also designed as a mixture of highly water-soluble odorants. Mixes E and F were designed to contain odorants that have been reported to stimulate cAMP and $\mathrm{IP}_{3}$ pathways, respectively (Sklar et al., 1986; Breer and Boekhoff, 1991; Restrepo et al., 1993). Stimulus solutions were prepared as $1 \mathrm{~mm}$ stocks in Ringer's solution and diluted to a final concentration of $50 \mu \mathrm{M}$ for each odorant in the solution. This stimulus concentration is well within the range $(1 \mathrm{nM}-1 \mathrm{~mm})$ of those used in previous studies on dissociated mammalian ORNs (Restrepo et al., 1993; Sato et al., 1994; Tareilus et al., 1995). Stocks were vortexed and sonicated before and after dilution to ensure that all components were in solution.

Optical recordings were obtained using an inverted microscope with a CCD video camera coupled to an image intensifier; the light source was a $75 \mathrm{~W}$ xenon lamp attenuated with neutral density filters. ORNs labeled with RH414 and/or green microspheres could be viewed simultaneously using a long-pass fluorescein filter set. Dual-excitation calcium imaging was performed using a standard fura-2 filter set (Omega Optical, Brattleboro, VT). Data were acquired every $4 \mathrm{sec}$; the average pixel values over the cell bodies of selected cells were calculated at 340 and $380 \mathrm{~nm}$ excitation, and ratios were obtained. Filter wheel control and image acquisition were performed using software designed in our laboratory.

Ratiometric imaging controlled for occasional movement artifacts and allowed us to estimate the magnitude of the stimulus-induced $\left[\mathrm{Ca}^{2+}\right]_{\text {i }}$ changes in a sample of responsive ORNs. $\left[\mathrm{Ca}^{2+}\right]_{\mathrm{i}}$ was estimated from the ratios using standard methods (Grynkiewicz et al., 1985). The $K_{\mathrm{d}}$ of fura- 2 for $\mathrm{Ca}^{2+}$ was taken to be $220 \mathrm{nM} ; R_{\max }$ and $R_{\min }$ were determined in intact cells by adding $5 \mu \mathrm{M}$ ionomycin in Ringer's solution $(1 \mathrm{mM}$ $\mathrm{Ca}^{2+}$ ) to saturate the fura- 2 and by subsequently bathing the cell in low- $\mathrm{Ca}^{2+}$ Ringer's solution supplemented with 5 mM EGTA. The mean resting $\left[\mathrm{Ca}^{2+}\right]_{\mathrm{i}}$ was $68 \pm 6 \mathrm{nM}($ mean $\pm \mathrm{SEM} ; n=4)$, and the peak responses to odorants were within the $400-600 \mathrm{~nm}$ range. These numbers should be considered approximate estimates of absolute $\left[\mathrm{Ca}^{2+}\right]_{i}$, because the calibration is prone to several errors, including variation in the apparent $K_{\mathrm{d}}$ of fura- 2 attributable to $\mathrm{pH}$ and ionic strength as well as incomplete equilibration of internal and external $\mathrm{Ca}^{2+}$ using ionomycin.

Dissociated ORNs were identified if they were retrogradely labeled and exhibited a fast onset, rapidly recovering $\mathrm{Ca}^{2+}$ response to $\mathrm{KCl}$ depolarization. Many traced ORNs retained characteristic morphology, including a dendrite and cilia; however, the tendency was for ORNs to retract their dendrites, forming a bowling pin-like shape. Unlabeled cells were considered to be ORNs based on morphological criteria and because they showed fast $\mathrm{KCl}$ and odorant-induced $\mathrm{Ca}^{2+}$ transients.

Analysis. All responses were repeated at least once; we took the largest response to a given stimulus to include in the analysis. Responses were normalized to the $\mathrm{KCl}$ response within cells to control for imaging artifacts present in some recordings. Stepwise logistic regression was used for pair-wise comparisons among the four groups [DM, DL, dorsal (D), and unlabeled]. This procedure tests for differences among populations in which the individual members are defined by a set of variables, in this case the normalized response amplitudes to each of the six mixtures. The analysis finds the combination of independent variables that best discriminate between two groups and estimates the probability that the groups differ. Significant differences are reported along with the variables (odorant mixtures) included in the model. Data were analyzed using SPSS 8.0 software and plotted using Mathematica 3.0 software (Wolfram Research). 


\section{Odorant Mixtures}

$\operatorname{Mix} A$

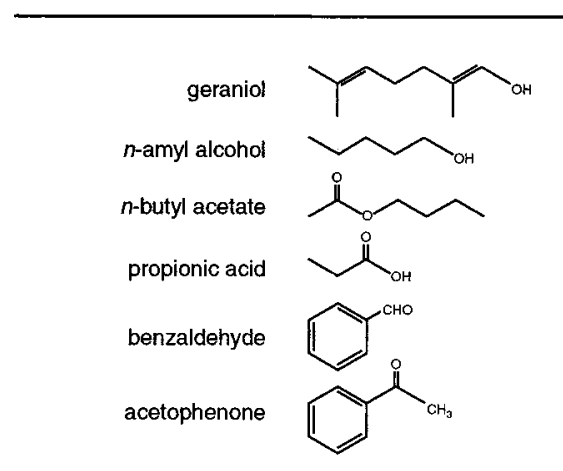

Mix C

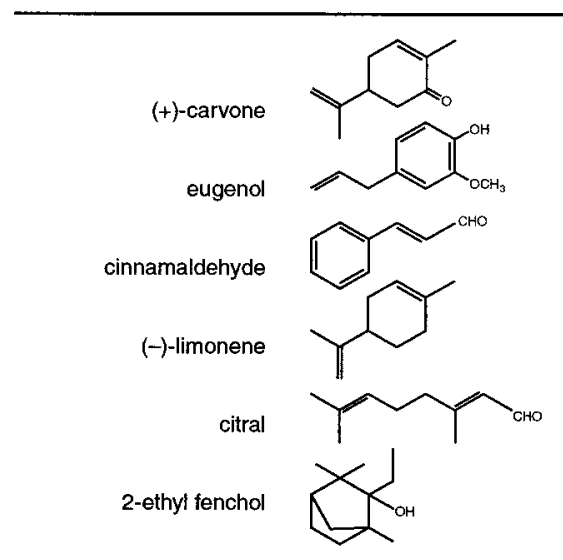

Mix E (CAMP)

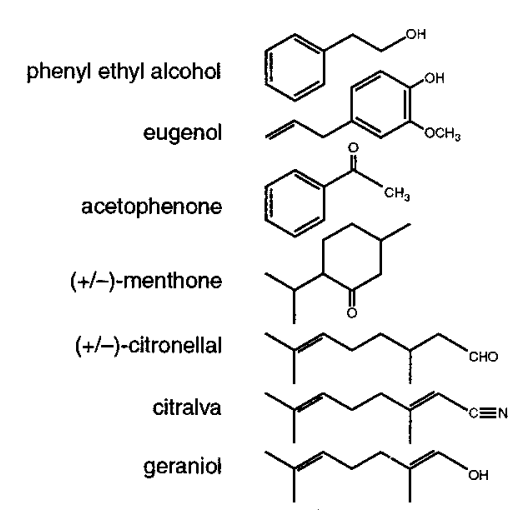

Mix B

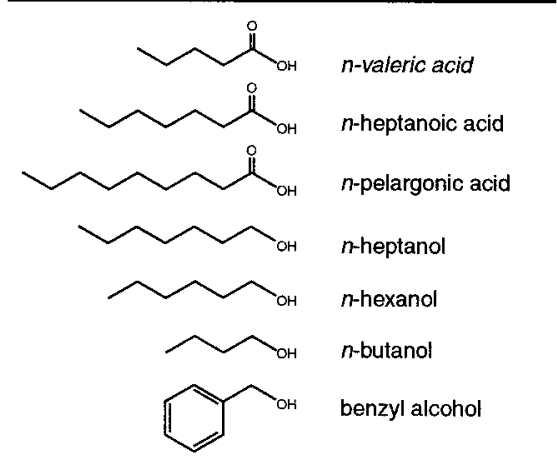

$\operatorname{Mix} \mathrm{D}$

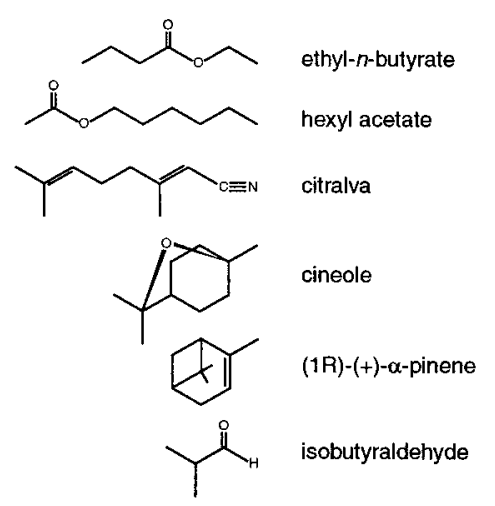

$\operatorname{Mix} F\left(I P_{3}\right)$

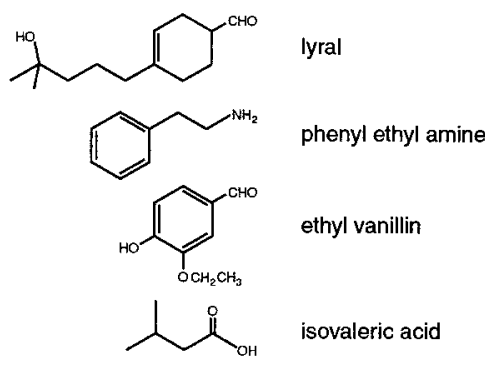

Figure 1. Molecular composition of the six odorant mixtures $(A-F)$. Each mixture was designed to include many different classes of molecules (see Materials and Methods). Mix E and Mix $F$ are mixtures of molecules that have been reported to increase cAMP and $\mathrm{IP}_{3}$, respectively (Sklar et al., 1986; Breer and Boekhoff, 1991). Four of the molecules in Mix $E$ are also found in other mixtures: geraniol and acetophenone are in $A$ and $E$, eugenol is in $C$ and $E$, and citralva is in $D$ and $E$.

\section{RESULTS}

\section{Retrograde tracing from visualized groups of glomeruli}

Focal injections of fluorescein-labeled microspheres were made into clusters of visualized glomeruli in the DM or DL OB (Fig. $2 A, B$, insets). DM injections typically involved $\sim 20$ glomeruli, and DL injections involved $\sim 30$ glomeruli (see Materials and Methods); this represents $2-3 \%$ of the total number of glomeruli ( 1000) in 2-week-old CF-1 mice (Pomeroy et al., 1990). Smaller injections (one to four glomerular widths) were made in preliminary experiments but resulted in too few labeled ORNs to make physiological recordings feasible. Injections were localized using the anterior and medial edges of the craniotomy as landmarks, and care was taken to inject the same region of the DM or DL OB across animals (see Materials and Methods). The width of the OB is $\sim 1.8 \mathrm{~mm}$ at this age, and DL and DM sites were separated by $\sim 1 \mathrm{~mm}$ (Fig. 2A,B, insets, $E, F$ ).

The microspheres were sufficiently bright to allow the tracing patterns to be observed in whole mounts of the septum and turbinates (DM, $n=12$; DL, $n=8$ ). Microsphere-labeled ORNs that were retrogradely traced from the two sites (DM-projecting and DL-projecting ORNs) were found in distinct but overlapping regions of the septum and turbinates of the OE (Fig. $2 A-D$ ). 

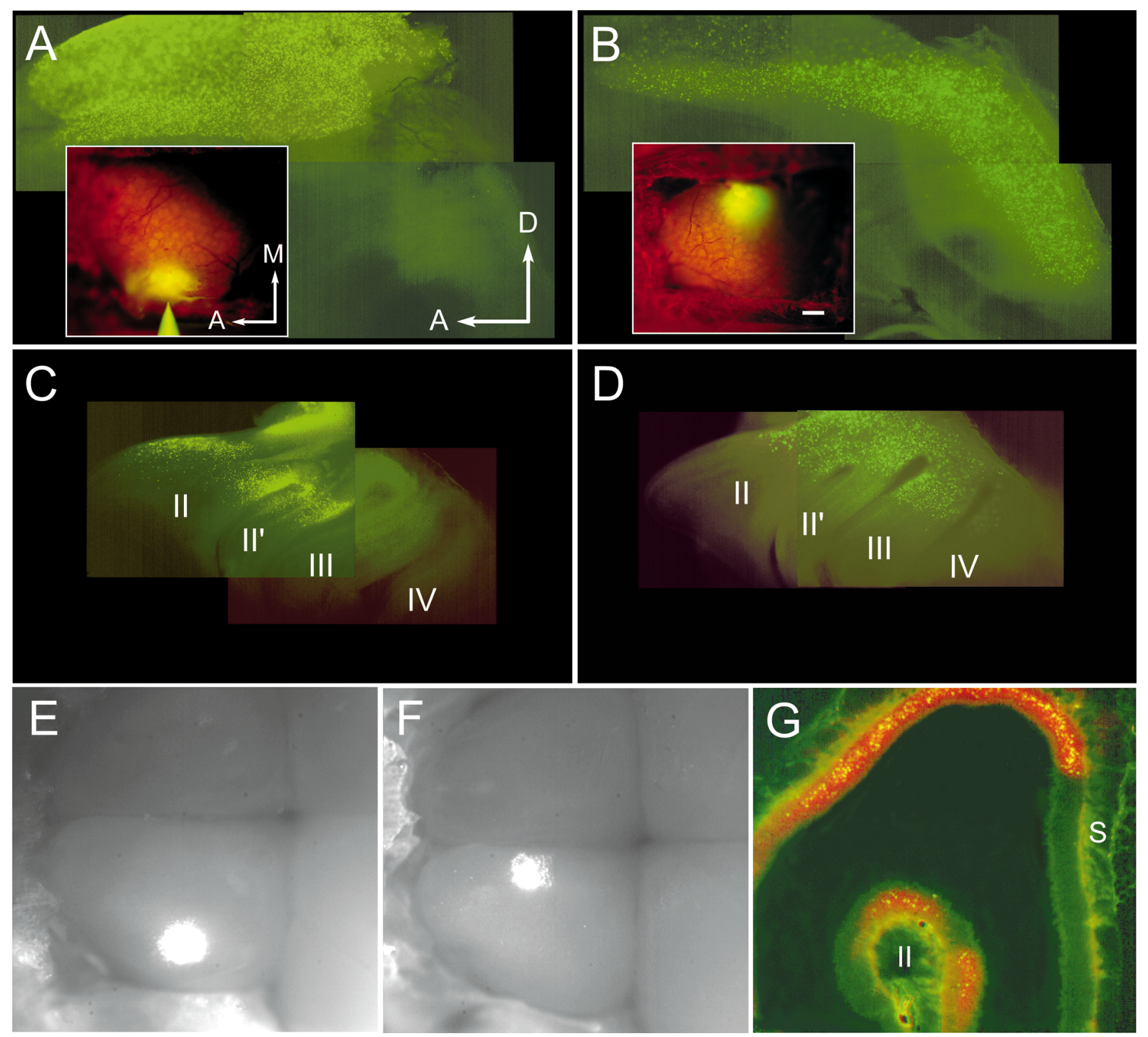

Figure 2. Distribution of retrogradely labeled ORNs in the OE. $A, B$, insets, Fluorescence images of the dorsal OBs of living mice taken immediately after injection of fluorescein-labeled latex microspheres (yellow) into the DL $(A)$ and DM $(B)$ sites. Glomeruli are seen (orange) in the regions stained with RH414. $A-D$, Whole mounts of the septum $(A, B)$ and the medial face of the turbinates $(C, D)$ show the distribution of microsphere label $4 \mathrm{~d}$ after injection. The anterior lateral $\mathrm{OE}$ is reflected dorsally in $A$ and $B$. Turbinates are numbered $I I-I V$ in $C$ and $D$. DL labeling was detectable in the anterior septum $(A)$ and in turbinates $I I$ and $I I^{\prime}(C)$. DM labeling formed an anterior to posterior band on the septal OE $(B)$ and in the dorsal turbinates $(D)$. Labeling in turbinate $I V$ in $D$ is out of the place of focus. $E, F$, Combined fluorescence and bright-field video images of both OBs exposed $2 \mathrm{~d}$ after injection with microspheres. In these images anterior is left, and the DL $(E)$ and DM $(F)$ injection sites are shown as bright spots in the left OBs. $G$, Coronal 20- $\mu$ m-thick section through the nasal cavity showing microspheres (yellow) and RH414 (orange) label in the OE $3 \mathrm{~d}$ after a large injection into the dorsocentral OB. $A$, Anterior; $M$, medial; $S$, septum. Scale bar: $A-D$, insets, $200 \mu \mathrm{m} ; E, F, 230 \mu \mathrm{m} ; G, 100 \mu \mathrm{m}$.

DL-projecting ORNs were found exclusively in the anterior dorsal recess and anterior turbinates II and II' (Fig. 2A,C). These data are consistent with patterns observed with injections into the DL quadrant of the OB in rat and hamster (Astic and Saucier, 1986; Astic et al., 1987; Schoenfeld et al., 1994). In contrast, DM-projecting ORNs were found in a stripe occupying the entire anterior to posterior extent of the dorsal septum and turbinates (Fig. 2B,D). Interestingly, an anteroposterior band of labeling was only observed if the retrograde tracer had access to the extreme medial edge of the OB. Injections placed $\sim 400 \mu \mathrm{m}$ lateral to the DM site resulted in a DL pattern of labeling or in a DL pattern in which a small fraction of the total label could be found in the caudal OE ( $n=3$; data not shown). Thus the DM site seems to be close to a transition point in the anatomical projection patterns between the $\mathrm{OB}$ and $\mathrm{OE}$ (Schoenfeld et al., 1994).

Surprisingly, RH414, which was used to visualize glomeruli, also acted as a retrograde tracer (Tsau et al., 1996) and could be observed in whole mounts (data not shown) and sections of the OE (Fig. $2 G$ ). Sections of OE and OB clearly demonstrated that 
RH414 stained only the dorsal half (and preferentially the DL surface) of the OB (data not shown). This was likely attributable to differential access of the dye to regions of the bulb through the dura, because breaching the dura with high-osmolarity treatment resulted in more uniform staining of the dorsal OB (LaMantia et al., 1992). The tracing pattern observed with RH414 was almost identical to the DL projection pattern seen with the smaller microsphere injections. However, within the labeled regions, many more ORNs were labeled with RH414 than with microspheres. This fortuitous result allowed us to use RH414 as a marker for ORNs that project to a large region of the D half of the OB.

\section{Responses of traced ORNs to odorant mixtures}

Dissociated ORNs were loaded with fura-2 for measuring odorant-induced calcium responses (Restrepo et al., 1990). The stimuli were a set of six odorant mixtures (mixes A-F), each of which was designed to include molecules with disparate chemical structures (see Materials and Methods, Fig. 1). We wished to test for response biases of ORNs based on where they project to the OB without making a priori assumptions as to which molecules might stimulate the different anatomical groups. The use of mixtures permitted initial screening of ORNs with many structurally diverse odorants using relatively few stimulus applications. After initial characterization, the mixtures were then broken down to identify individually effective stimuli.

DM- or DL-projecting microsphere-labeled ORNs were easily identified in the dissociated preparations by bright green, punctate fluorescence (Fig. 3A,B), whereas the D-projecting, RH414labeled ORNs were identified by red, punctate fluorescence (data not shown). We recorded from 200 retrogradely labeled ORNs, each of which was stimulated with $\mathrm{KCl}(100 \mathrm{~mm})$ to assess viability and subsequently tested with all six mixtures. Of these, 12 of $33(36.4 \%)$ D-projecting, 21 of 99 (21.2\%) DL-projecting, and 20 of $68(29.4 \%)$ DM-projecting ORNs responded to at least one of the mixtures. Stimulation with $\mathrm{KCl}$ or odorants elicited short-latency increases in $\left[\mathrm{Ca}^{2+}\right]_{\mathrm{i}}$ that typically lasted $1-2 \mathrm{~min}$ (Fig. 3C). The durations of the odorant responses were similar and in some cases shorter than the somatic $\mathrm{Ca}^{2+}$ transients seen in previous studies of dissociated ORNs (Restrepo et al., 1993; Sato et al., 1994; Tareilus et al., 1995; Leinders-Zufall et al., 1997) (see Discussion).

We first asked whether selecting ORNs that project to different discrete regions of the dorsal OB biases the responses to our stimulus set. DM- and DL-projecting ORNs exhibited markedly different response profiles, preferentially responding to different odorant mixtures (Fig. 4, compare $A, B$ ). Overall, the DL group responded most often to mixes $\mathrm{C}$ and $\mathrm{E}$, with the most common response profile being “A, C, E." In fact, there was a significant correlation between $\mathrm{C}$ and $\mathrm{E}$ responses (Pearson correlation coefficient, $r=0.613$; $p<0.01$ ). We reasoned that this might be caused by the fact that mixes $\mathrm{C}$ and $\mathrm{E}$ both contain eugenol (see below). In contrast, the $\mathrm{DM}$ group responded best to mix $\mathrm{B}$, with the most common profile being " $\mathrm{B}$ " only. The most striking differences between the two groups were that responses to mixes $\mathrm{C}$ and $\mathrm{E}$ observed in the DL population were completely absent from the DM population and that the number of responses to mix B observed in the DM population was significantly higher than in the DL population (Fisher's exact test, $p<0.001$ ). The two populations could be separated with respect to $\mathrm{C}$ and $\mathrm{E}$ responsiveness (logistic regression analysis, $p<0.001$ ). These data demonstrate directly that individual ORNs that are partially
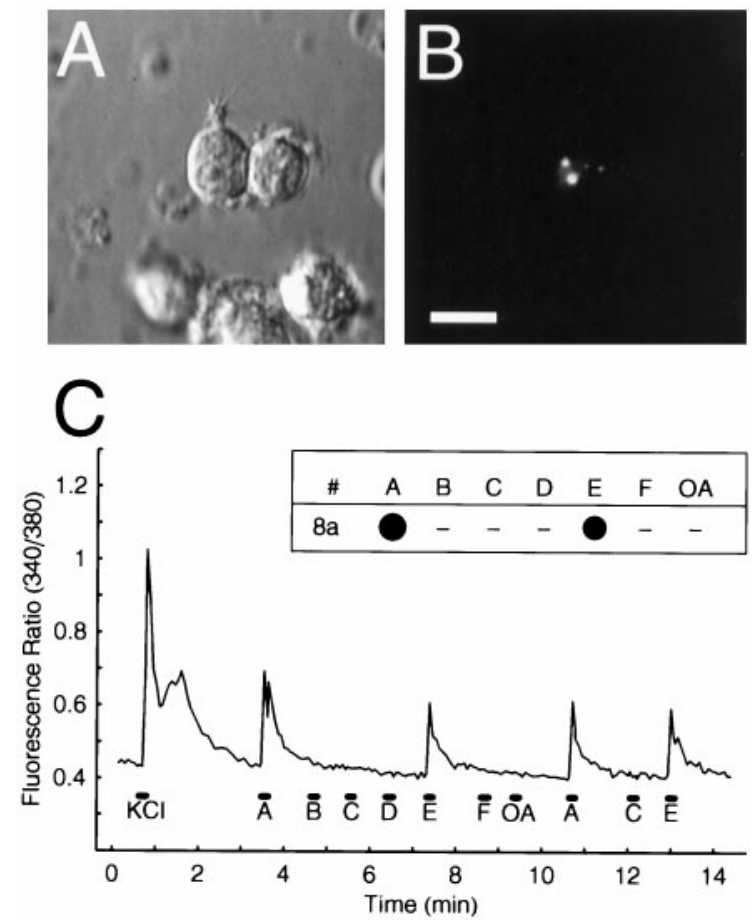

Figure 3. Odorant responses of a retrogradely labeled ORN tested with odorant mixtures. $A$, Dissociated odorant-responsive ORN viewed under Nomarsky optics showing a short dendrite and cilia. $B$, Fluorescence image of the same field showing the microsphere labeling (white dots). Microsphere-labeled ORNs were often but not always labeled with RH414. Scale bar: $A, B, 10 \mu \mathrm{m}$. $C$, Fura-2 measurements in a single retrogradely labeled ORN (dorsolateral \#8a in Fig. 4A) stimulated with 10 sec pulses (black bars) of six odorant mixtures $(A-F)$ and an OA mix, as well as with a $4 \mathrm{sec}$ pulse of $\mathrm{KCl}$. Inset, "Dot" plot of the odorant response profile derived from the analog data for this cell. The dot area represents the response amplitude as a percentage of the $\mathrm{KCl}$ response; the largest responses observed to each mixture were used for these plots. Dashes indicate no response. This cell responded to mixes A and E.

intermingled in the $\mathrm{OE}$ and that project to two different loci in the OB exhibit different response profiles to the same stimulus set.

We next asked whether the response properties of DM- or DL-projecting ORNs were distinguishable from those of ORNs projecting to more broad regions of the OB. These included RH414-labeled, D-projecting ORNs and 17 unlabeled ORNs, which likely project to regions of the OB (ventral half) not exposed to either RH414 or microspheres (Fig. 4C,D). Responses seen in the DL-projecting ORNs were not statistically different from responses in 12 D-projecting ORNs but were significantly different from 17 unlabeled ORNs with respect to $\mathrm{D}$ and $\mathrm{E}$ responses (logistic regression, $p<0.001$ ). Thus, with respect to this stimulus set, responses of ORNs that project to a small DL region of the $\mathrm{OB}$ were indistinguishable from those projecting to a more broad dorsal OB region.

In contrast, DM-projecting ORNs were separable from both D-projecting ( $p<0.001$, with respect to $\mathrm{C}$ and $\mathrm{B}$ responses) and unlabeled ORNs ( $p<0.001$, with respect to $\mathrm{C}$ and $\mathrm{D}$ responses). Interestingly, when compared with the other three groups, DM neurons exhibited the least variability in the number of different profiles per sample size, implying that this group is the most homogeneous of all the samples. This holds true when comparing populations of ORNs that project to similar numbers of glomeruli; for DL-projecting ORNs $(n=21)$ there were 12 different 

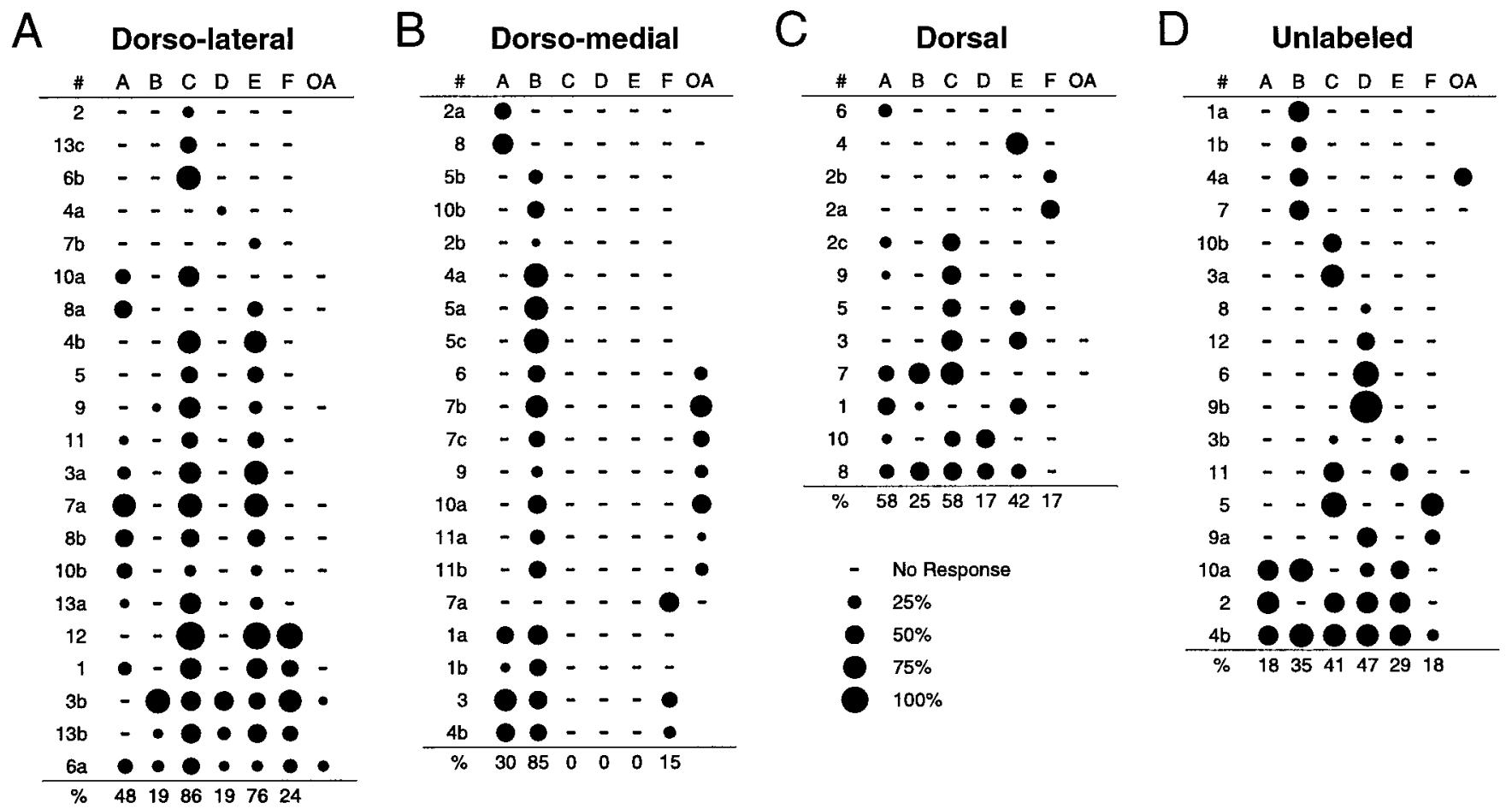

Figure 4. Odorant response profiles for ORNs responsive to the odorant mixtures. $A$, DL-projecting microsphere-labeled ORNs $(n=21)$. $B$, DM-projecting microsphere-labeled ORNs $(n=20)$. $C$, RH414-labeled, D-projecting ORNs $(n=12)$. $D$, Unlabeled ORNs $(n=17)$. Each row shows the responses of a single ORN to the six mixtures $(A-F)$ as well as to the OA mix, as in Figure $3 C$. Blank spaces indicate that the stimulus was not tested, and dashes indicate no response. The percentage of responsive cells responding to each mixture is indicated below each plot. Cell identification numbers are to the left; within each panel, cells with the same number are from the same animal. Cells are ordered by increasing breadth of response.

response profiles with respect to the six mixtures, although for DM-projecting ORNs $(n=20)$ there were only five profiles (Fig. $4 A, B)$. These data suggest that the homogeneity of convergent ORNs may vary for different regions of the OB.

The response profiles of DM cells consisted exclusively of responses to mixtures containing organic acids (mixes $\mathrm{A}, \mathrm{B}$, and F). This led us to ask whether DM-projecting ORNs respond preferentially to the acids in our stimulus set. As a first step in testing this, 18 ORNs stimulated with mixes A-F were also tested with an organic acids (OA) mixture containing only the acids from the original six mixtures. Seven of nine DM-projecting and two of nine DL-projecting ORNs responded to the OA mix (Fig. $4 A, B)$. These data suggest that a higher proportion of DMprojecting ORNs responds to the organic acids in the mixtures compared with DL-projecting ORNs.

\section{Responses of traced ORNs to mixture components}

To identify individual odorants that activate ORNs, we tested cells with components of the individual mixtures. Because a majority of responsive DL-projecting ORNs $(86 \%)$ responded to mix $\mathrm{C}$, we sought to identify the molecular species in mix $\mathrm{C}$ that stimulated this group. To do this, we performed an experiment in which another 92 DL-projecting ORNs were stimulated with mix $\mathrm{C}$ and its components (carvone, eugenol, cinnamaldehyde, limonene, citral, and ethyl-fenchol) as well as geraniol and acetophenone (Fig. 5A). The latter two stimuli are components of mixes $\mathrm{A}$ and $\mathrm{E}$ that we reasoned DL-projecting ORNs might respond to given the prevalence of $\mathrm{A}, \mathrm{C}, \mathrm{E}$ profiles in the mixture data. In this experiment, 19 cells responded to at least one stimulus, and 17 responded to mix C. Of the 17 mix C-responsive ORNs, 9 responded to carvone, eugenol, or cinnamaldehyde only, 5 responded to more than one of the individual mix $\mathrm{C}$ components, and 3 did not respond to any of the components (Fig. 5C). There were no responses observed with limonene, geraniol, or ethyl-fenchol, and mix C-responsive ORNs typically did not respond to the mix A and E components geraniol and acetophenone. Interestingly, responses to a given molecule (carvone, for instance) were observed in the context of a number of different response profiles, providing a clear demonstration that, within this anatomically restricted set of ORNs, a given odorant induces responses in cells with different but overlapping response properties.

For the DM group, we had shown that mix B was the most effective stimulus, eliciting responses in $85 \%$ of the responsive ORNs. As a result, a new group of 98 DM-projecting ORNs was stimulated with mix B and its component odorants (valeric acid, heptanoic acid, pelargonic acid, heptanol, hexanol, butanol, and benzyl-alcohol) and mix $\mathrm{C}$ (Fig. $5 B$ ). In this experiment, 18 cells responded to at least one of the stimuli. Of 17 mix B-responsive cells, 16 responded to at least one of the individual organic acids, and 14 responded to the acids but not the alcohols (Fig. 5D).

It has been shown previously that ORNs isolated from mouse septal OE respond to carboxylic acids and alcohols of similar carbon chain length, but that a majority do not discriminate acids from alcohols when tested over a range of concentrations (Sato et al., 1994). Interestingly, $88 \%$ of the acid-responsive DMprojecting ORNs, which lie in a similar region of the septal OE, 

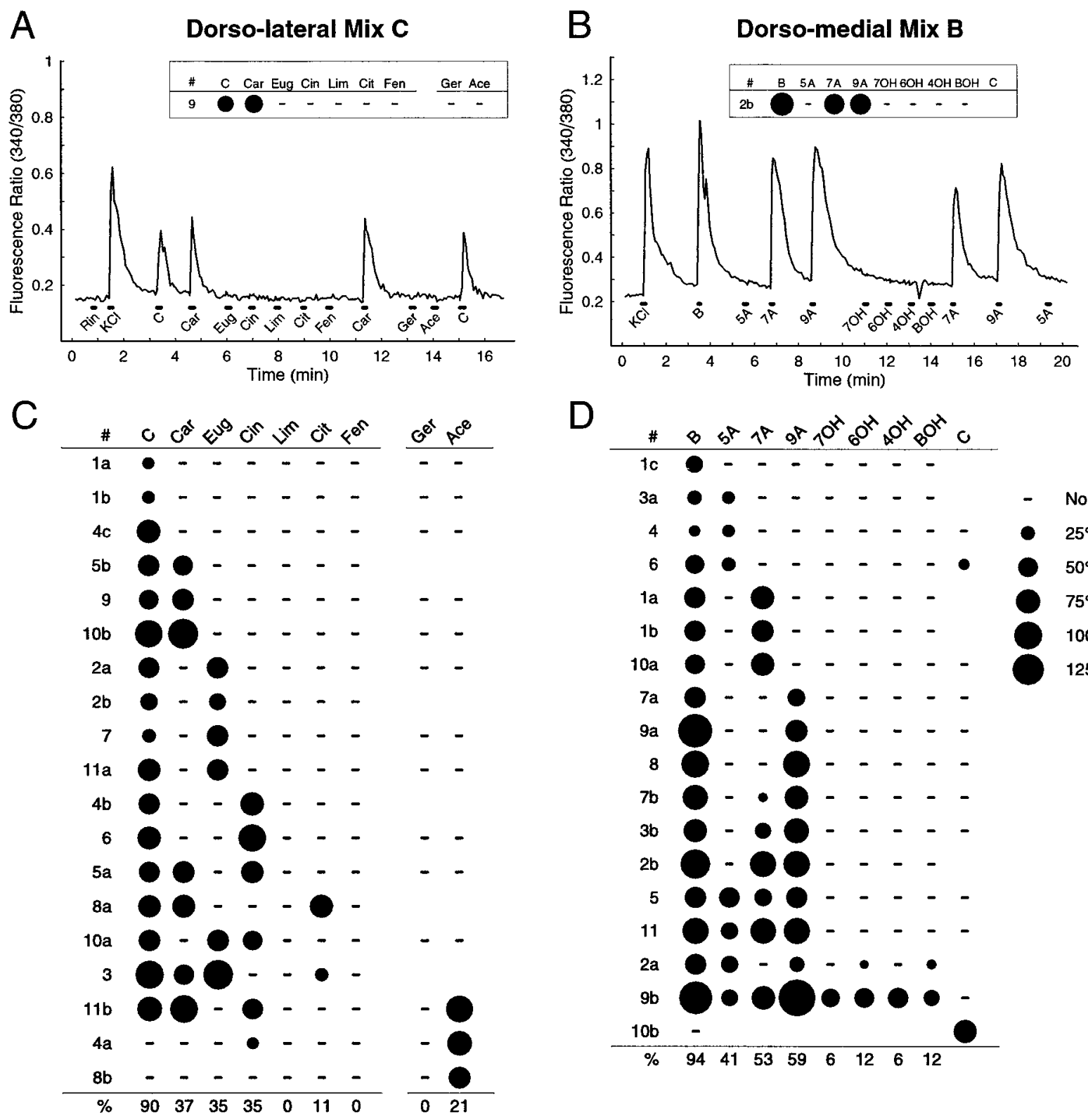

$\mathrm{D}$

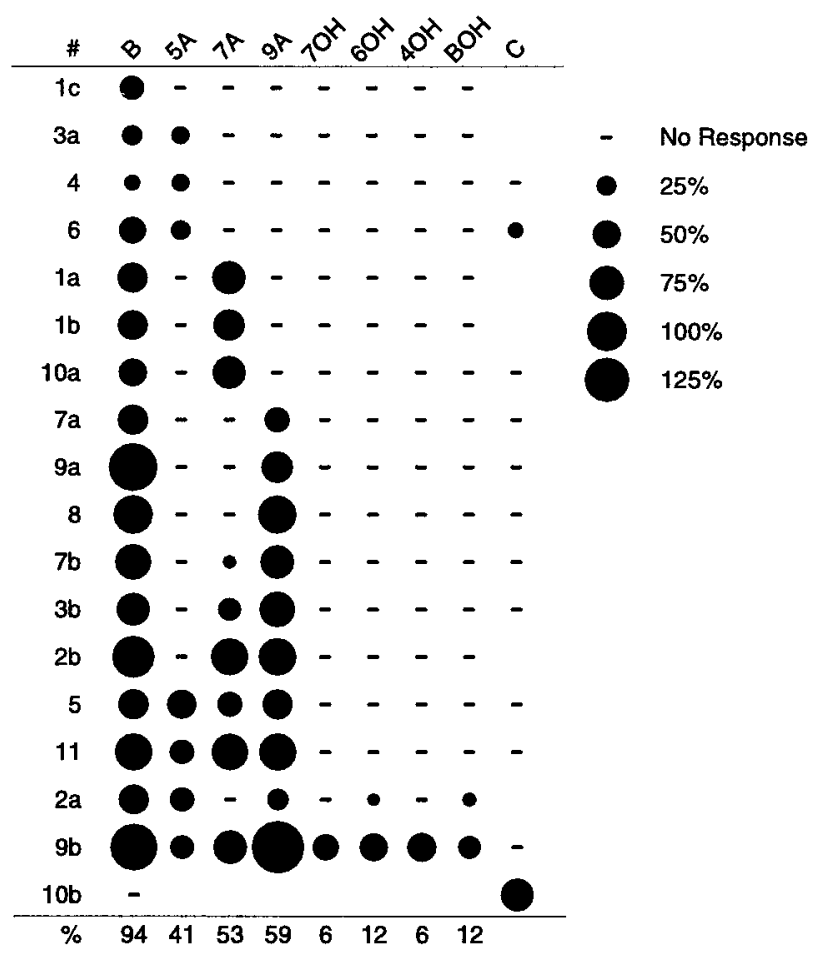

Figure 5. Odorant responses of retrogradely labeled ORNs to individual mixture components. $A$, Calcium responses from a single DL-projecting ORN tested with mix C and its components, (+)-carvone (Car), eugenol (Eug), cinnamaldehyde (Cin), (-)-limonene ( Lim), citral (Cit), and 2-ethyl fenchol $(\mathrm{Fen})$ and with geraniol (Ger) and acetophenone (Ace), two components of mixes A and E. Rin and $\mathrm{KCl}$ mark stimulations with normal and high-K ${ }^{+}$ Ringer's solution, respectively. $B$, Calcium responses from a single DM-projecting ORN tested with mix B and its components, $n$-valeric acid ( $5 A$ ), $n$-heptanoic acid $(7 A), n$-pelargonic acid $(9 A), n$-heptanol $(7 O H), n$-hexanol $(6 O H), n$-butanol $(4 O H)$, and benzyl alcohol $(B O H)$ and with mix C. Dot response profiles for the two cells are shown in the insets. The small dip in the ratio after $4 O H$ in $B$ is an optical artifact. $C, D$, Summary of response profiles (as in Fig. 4) for 19 DL-projecting and 18 DM-projecting ORNs. Blank spaces indicate that the stimulus was not tested, and dashes indicate no response.

discriminated the acids from alcohols of similar carbon chain length at the single concentration used here. Most strikingly, of the nine heptanoic acid-responsive neurons, only one responded to heptanol. Although it is possible that higher stimulus concentrations might have induced alcohol responses in these ORNs, our stimulus concentrations were already at the high end of those that might be encountered under physiological conditions.

Taken with the results using mixtures, these data show that a majority of responsive DM-projecting ORNs respond to similar organic acids in our stimulus set. Thus, the DM OB likely receives a large proportion of its inputs from organic acid-responsive ORNs.

\section{DISCUSSION}

The goal of the present study was to characterize the spatial representation of odorant information in the projections between the $\mathrm{OE}$ and the $\mathrm{OB}$ in a species in which there are anatomical and OR gene expression data. Our results provide direct evidence that ORNs projecting to two regions of the dorsal OB respond 
best to individual molecules that are structurally different, and that these regions appear to differ in the homogeneity of their response profiles. The data also indicate that a subset of distributed ORNs that converge onto a small region of the DM OB can be shown to respond to odorants with similar chemical structures. These findings are consistent with molecular data showing that ORNs expressing the same OR gene converge onto glomeruli in the OB. Moreover, given the lack of information on the relationship between the OR genes and the ligand-binding properties of their respective proteins (Zhao et al., 1998), these studies provide a view of the functional topography of the olfactory system not provided by previous molecular investigations.

\section{Retrograde tracing patterns}

Several lines of evidence suggest that there may be a mediolateral symmetry to the organization of the mammalian OB. ORNs that express the same OR gene converge onto medial and lateral glomeruli (Ressler et al., 1994; Vassar et al., 1994; Mombaerts et al., 1996), and the medial and lateral "hemispheres" of the OB are connected by an intrabulbar association pathway connecting homotopic regions of the OB (Schoenfeld et al., 1985). However, the true mediolateral and dorsoventral axes of the $\mathrm{OB}$ are thought to be rotated medially about the anteroposterior axis (Schoenfeld et al., 1985, 1994). With respect to these "corrected" axes, our DL site is within the lateral half of the OB, while our DM site is located at or near the true dorsal meridian. Thus, DM injections likely labeled ORNs projecting to both the medial and lateral hemispheres, whereas DL injections likely labeled only ORNs projecting to the lateral hemisphere. The DM and DL tracing patterns were strikingly different but did overlap considerably in the anterior OE. The DM pattern resembled the anteroposterior stripe of OR gene expression in the dorsal-most OE region ("zone 1") observed in in situ hybridization studies (Ressler et al., 1993; Vassar et al., 1993). In contrast, the DL pattern formed only the rostral half of a more ventrally located stripe. These data support the idea that a complement of both medially and laterally projecting ORNs is required to produce a complete anteroposterior stripe of ORNs on either the septum or turbinates (Schoenfeld et al., 1994; Mombaerts et al., 1996).

\section{Response properties of retrogradely traced ORNs}

Our experiments required the use of enzymes to dissociate ORNs. The cells included in our analyses maintained normal physiological $\left[\mathrm{Ca}^{2+}\right]_{\mathrm{i}}$, exhibited robust calcium transients to $\mathrm{KCl}$ and odorant stimulation, and responded differentially to odorants. These observations attest to the health of the neurons. Our findings are consistent with studies showing that somatic $\mathrm{Ca}^{2+}$ responses typically last 1-2 min after brief odorant or IBMX stimulation (Restrepo et al., 1993; Sato et al., 1994; Tareilus et al., 1995; Leinders-Zufall et al., 1997). Thus, the time course of our $\mathrm{Ca}^{2+}$ responses is unlikely to be attributable solely to stimulus washout time but may also reflect intrinsic $\mathrm{Ca}^{2+}$ dynamics in ORNs (e.g., $\mathrm{Ca}^{2+}$ release from intracellular compartments; Leinders-Zufall et al., 1997).

There are few studies that have characterized the response breadth of individual mammalian ORNs using broad classes of odorants (Sicard, 1986). Data from amphibians show that the response selectivity of ORNs varies considerably (Duchamp et al., 1974; Getchell, 1974; Revial et al., 1978, 1982). Similarly, in the present experiments, some mouse ORNs responded to stimuli with quite different structures, whereas others displayed a remarkable ability to discriminate among molecules that differed by small changes in carbon chain length or functional group (Sato et al., 1994). Thus, as in other species, odorant information in the mouse is likely encoded by populations of variably selective ORNs.

Our view of the tuning characteristics among groups of traced ORNs may be dependent on the odorant set used. This is illustrated by the fact that $\sim 75 \%$ of the KCl-responsive ORNs we tested failed to respond to odorants, either because they were damaged by the dissociation procedure or were not presented with an adequate stimulus. Thus, some undefined proportion of potentially responsive cells were not characterized, because they did not respond to any of the odorants presented. Therefore, the data represent one view of a subpopulation of anatomically defined ORNs selected by responsiveness to this particular odorant set. We consider it significant, however, when starting with a diverse odorant set chosen more or less randomly, that prominent response differences among anatomically selected ORNs emerged. This suggests that the overall differences in responsiveness between the DM and DL sites is robust and can likely be detected using a number of stimulus sets.

The assessment of variability between groups of traced ORNs is likely to be influenced strongly by the choice of stimuli and by variability in injection sizes and locations. It is worth noting that DL injections involved a few more glomeruli and varied more in location across animals than did DM injections. Consequently, the differences in heterogeneity between the groups may be related to differences in variability in placing the injection sites. Both sets of injections, however, were similar with respect to percentage of the entire glomerular sheet labeled.

Another possible difference might be in the developmental stages of ORNs projecting to the DM and DL regions in young animals. This would appear less likely for several reasons. The available data suggest that ORNs are distributed in adult-like patterns and express molecules involved in odorant transduction before birth (Margalit and Lancet, 1993; Menco et al., 1994; Sullivan et al., 1995), and that potential effects of immaturity on odorant responsiveness are gone by embryonic day 19 in rats (Gesteland et al., 1982). In addition, medially and laterally projecting neurons expressing the P2 OR gene are qualitatively similar in P0 and adult animals at the gross morphological level (Mombaerts et al., 1996), and ORN terminals within glomeruli at this time are also morphologically indistinguishable from adults (Klenoff and Greer, 1998). If the DM population is in fact more homogeneous, an interesting conjecture is that the homogeneity of ORNs projecting to groups of glomeruli may vary with location relative to natural anatomical boundaries in the $\mathrm{OB}$ (see above).

\section{Functional topography of the mammalian OB}

Much of what we know about the distribution of odorant-induced activity across the glomerular layer in mammals has come from summed activity measures or from recordings of $\mathrm{OB}$ output neurons. Our data are the first to address this issue at the level of individual ORNs. Studies of 2-deoxyglucose uptake in rats have demonstrated a DM focal region of bulbar activation after stimulation with propionic acid (Bell et al., 1987), a component of mix A. However, DM-projecting ORNs were not preferentially responsive to mix A as might be expected. Although it is possible that a high proportion of propionic acid-responsive cells project to regions near the DM site, this will have to be tested explicitly. Our data fit well with single-unit recordings from rabbit mitral and tufted cells showing that neurons in the DM OB are activated in response to longer-chain carboxylic acids (and aldehydes) but 
not alcohols of similar carbon chain length (Imamura et al., 1992; Mori et al., 1992). Our data also provide support for the hypothesis that the response bias of DM-situated mitral cells may be attributed to direct input from organic acid-responsive ORNs. Unlike for the DM OB, the odorant response properties of individual mitral cells in the DL OB are not known. Our studies have identified a set of molecules (carvone, eugenol, and cinnamaldehyde) to which mitral cells in the DL mouse OB may be preferentially responsive. The correspondence between our data from young mice and these data from adult rabbits suggests that the response biases observed in both studies may reflect a general feature of the organization of mammalian OBs.

\section{Functional convergence of ORN afferents}

DM-projecting ORNs were clearly biased toward responding to the organic acids in our stimulus set. It is important to realize that the data do not demonstrate that DM-projecting ORNs respond only to organic acids for several reasons. First, as noted above, some proportion of the unresponsive ORNs may have responded to odorants that were not included in our stimulus set. Second, the data show that two DM-projecting ORNs did not respond to acids (Fig. 4B, \#8, \#7a) and four DM-projecting ORNs also responded to nonacid stimuli (Fig. 5D, \#2a, \#9b, \#6, \#10b). Taken together, the data suggest that acid-responsive ORNs can synapse in close proximity to ORNs that respond to other types of molecules. Nonetheless, the prevalence of the acid-responsive profiles demonstrates that the DM OB is a site of convergence of a large proportion of acid-responsive ORNs. Because our injection sites encompass $\sim 20$ glomeruli, it is likely that several glomeruli in this region receive inputs from acid-responsive ORNs. These observations indicate that some groups of glomeruli receive afferents from ORNs that carry similar information (Friedrich and Korsching, 1997).

One of the operations that $\mathrm{OB}$ circuitry may perform on incoming afferent activity is a modification of inputs to neighboring glomeruli via lateral inhibition (Yokoi et al., 1995). It is generally thought that glomeruli may be functional units (Jourdan et al., 1980; Kauer, 1980, 1987; Lancet et al., 1982; Guthrie et al., 1993; Kauer and Cinelli, 1993; Shepherd, 1994; Mori, 1995), receiving inputs from ORNs with similar response properties. If this is true, a key issue in olfactory coding will be to understand how similar the response properties are of ORNs projecting to a single glomerulus or groups of glomeruli among which lateral interactions occur. This question bears directly on the kind of processing that takes place in local OB circuits.

\section{REFERENCES}

Astic L, Saucier D (1986) Anatomical mapping of the neuroepithelial projection to the olfactory bulb in the rat. Brain Res Bull 16:445-454.

Astic L, Saucier D, Holley A (1987) Topographical relationships between olfactory receptor cells and glomerular foci in the rat olfactory bulb. Brain Res 424:144-152.

Bell GA, Laing DG, Panhuber H (1987) Odor mixture suppression: evidence for a peripheral mechanism in human and rat. Brain Res 426:8-18.

Breer H, Boekhoff I (1991) Odorants of the same odor class activate different second messenger pathways. Chem Senses 16:19-29.

Cinelli AR, Hamilton KA, Kauer JS (1995) Salamander olfactory bulb neuronal activity observed by video rate, voltage-sensitive dye imaging: III. Spatial and temporal properties of responses evoked by odorant stimulation. J Neurophysiol 73:2053-2071.

Duchamp A, Revial MF, Holley A, MacLeod P (1974) Odour discrimination by frog olfactory neurons. Chem Senses 1:213-233.

Friedrich RW, Korsching SI (1997) Combinatorial and chemotopic odorant coding in the zebrafish olfactory bulb visualized by optical imaging. Neuron 18:737-752.
Gesteland RC, Yancey RA, Farbman AI (1982) Development of olfactory receptor neuron selectivity in the rat fetus. Neuroscience 7:3127-3136.

Getchell TV (1974) Unitary responses in frog olfactory epithelium to sterically related molecules at low concentrations. J Gen Physiol 64:241-261.

Grynkiewicz G, Poenie M, Tsien RY (1985) A new generation of $\mathrm{Ca}^{2+}$ indicators with greatly improved fluorescence properties. J Biol Chem 260:3440-3450.

Guthrie KM, Anderson AJ, Leon M, Gall C (1993) Odor-induced increases in c-fos mRNA expression reveal an anatomical "unit" for odor processing in olfactory bulb. Proc Natl Acad Sci USA 90:3329-3333.

Imamura K, Mataga N, Mori K (1992) Coding of odor molecules by mitral-tufted cells in rabbit olfactory bulb. I. Aliphatic compounds. J Neurophysiol 68:1986-2002.

Joerges J, Küttner A, Galizia G, Menzel R (1997) Representation of odours and odour mixtures visualized in the honeybee brain. Nature 387:285-288.

Jourdan F, Duveau A, Astic L, Holley A (1980) Spatial distribution of $\left[{ }^{14} \mathrm{C}\right] 2$-deoxyglucose uptake in the olfactory bulbs of rats stimulated with two different odours. Brain Res 188:139-154.

Katz LC, Iarovici AM (1990) Green fluorescent latex microspheres: a new retrograde tracer. Neuroscience 34:511-520.

Kauer JS (1980) Some spatial characteristics of central information processing in the vertebrate olfactory system. In: Olfaction and taste VII (van der Starre H, ed), pp 227-236. London: IRL.

Kauer JS (1987) Coding in the olfactory system. In: Neurobiology of taste and smell (Finger TE, Silver WL, eds), pp 205-231. New York: Wiley.

Kauer JS, Cinelli AR (1993) Are there structural and functional modules in the vertebrate olfactory bulb? Microsc Res Tech 24:157-167.

Klenoff JR, Greer CA (1998) Postnatal development of olfactory receptor cell axonal arbors. J Comp Neurol 390:256-267.

LaMantia AS, Purves D (1989) Development of glomerular pattern visualized in the olfactory bulbs of living mice. Nature 341:646-649.

LaMantia AS, Pomeroy SL, Purves D (1992) Vital imaging of glomeruli in the mouse olfactory bulb. J Neurosci 12:976-988.

Lancet D, Greer CA, Kauer JS, Shepherd GM (1982) Mapping of odorrelated neuronal activity in the olfactory bulb by high-resolution 2-deoxyglucose autoradiography. Proc Natl Acad Sci USA 79:670-674.

Leinders-Zufall T, Rand MN, Shepherd GM, Greer CA, Zufall F (1997) Calcium entry through cyclic nucleotide-gated channels in individual cilia of olfactory receptor cells: spatiotemporal dynamics. J Neurosci 17:4136-4148.

MacKay-Sim A, Kesteven S (1994) Topographic patterns of responsiveness to odorants in the rat olfactory epithelium. J Neurophysiol 71:397-398

MacKay-Sim A, Shaman P, Moulton DG (1982) Topographic coding of olfactory quality: odorant specific patterns of epithelial responsivity in the salamander. J Neurophysiol 48:584-596.

Margalit T, Lancet D (1993) Expression of olfactory receptor and transduction genes during rat development. Dev Brain Res 73:7-16.

Menco BPM, Tekula FD, Farbman AI, Danho W (1994) Developmental expression of G-proteins and adenylyl cyclase in peripheral olfactory systems. Light microscopic and freeze-substitution electron microscopic immunocytochemistry. J Neurocytol 23:708-727.

Mombaerts P, Wang F, Dulac C, Chao SK, Nemes A, Mendelsohn M, Edmondson J, Axel R (1996) Visualizing an olfactory sensory map. Cell 87:675-686.

Mori K (1995) Relation of chemical structure to specificity of response in olfactory glomeruli. Curr Opin Neurobiol 5:467-474.

Mori K, Mataga N, Imamura K (1992) Differential specificities of single mitral cells in rabbit olfactory bulb for a homologous series of fatty acid odor molecules. J Neurophysiol 67:786-789.

Pomeroy SL, LaMantia AS, Purves D (1990) Postnatal construction of neural circuitry in the mouse olfactory bulb. J Neurosci 10:1952-1966.

Ressler KJ, Sullivan SL, Buck LB (1993) A zonal organization of odorant receptor gene expression in the olfactory epithelium. Cell 73:597-609.

Ressler KJ, Sullivan SL, Buck LB (1994) Information coding in the olfactory system: evidence for a stereotyped and highly organized epitope map in the olfactory bulb. Cell 79:1245-1255.

Restrepo D, Miyamoto T, Bryant BP, Teeter JH (1990) Odor stimuli trigger influx of calcium into olfactory neurons of the channel catfish. Science 249:1166-1168. 
Restrepo D, Okada Y, Teeter JH (1993) Odorant-regulated $\mathrm{Ca}^{2+}$ gradients in rat olfactory neurons. J Gen Physiol 102:907-924.

Revial MF, Duchamp A, Holley A (1978) Odour discrimination by frog olfactory receptors: a second study. Chem Senses 3:7-21.

Revial MF, Sicard G, Duchamp A, Holley A (1982) New studies on odour discrimination in the frog's olfactory receptor cells. I. Experimental results. Chem Senses 7:175-190.

Royet JP, Souchier C, Jourdan F, Ploye H (1988) Morphometric study of the glomerular population in the mouse olfactory bulb: numerical density and size distribution along the rostrocaudal axis. J Comp Neurol 270:559-568.

Sato T, Hirono J, Tonoike M, Takebayashi M (1994) Tuning specificities to aliphatic odorants in mouse olfactory receptor neurons and their local distribution. J Neurophysiol 72:2980-2989.

Schoenfeld TA, Marchand JE, Macrides F (1985) Topographic organization of tufted cell axonal projections in the hamster main olfactory bulb: an intrabulbar association system. J Comp Neurol 235:503-518.

Schoenfeld TA, Clancy AN, Forbes WB, Macrides F (1994) The spatial organization of the peripheral olfactory system of the hamster: part I, Receptor neuron projections of the main olfactory bulb. Brain Res Bull 34:183-210.

Scott JW, Davis LM, Shannon D, Kaplan C (1996) Relation of chemical structure to spatial distribution of sensory responses in rat olfactory epithelium. J Neurophysiol 75:2036-2049.

Scott JW, Shannon DE, Charpentier J, Davis LM, Kaplan C (1997) Spatially organized response zones in rat olfactory epithelium. J Neurophysiol 77:1950-1962.

Shepherd GM (1994) Discrimination of molecular signals by the olfactory receptor neuron. Neuron 13:771-790.

Sicard G (1986) Electrophysiological recordings from olfactory receptor cells in adult mice. Brain Res 397:405-408.
Sklar PB, Anholt RRH, Snyder SH (1986) The odorant-sensitive adenylate cyclase of olfactory receptor cells. J Biol Chem 261: $15538-15543$.

Stewart WB, Pedersen PE (1987) The spatial organization of olfactory nerve projections. Brain Res 411:248-258.

Stewart WB, Kauer JS, Shepherd GM (1979) Functional organization of rat olfactory bulb analyzed by the 2-deoxyglucose method. J Comp Neurol 185:715-734.

Sullivan SL, Bohm S, Ressler KJ, Horowitz LF, Buck LB (1995) Targetindependent pattern specification in the olfactory epithelium. Neuron 15:779-789.

Tareilus E, Noe J, Breer H (1995) Calcium signals in olfactory neurons. Biochim Biophys Acta 1269:129-138.

Tsau Y, Wenner P, O'Donovan MJ, Cohen LB, Loew LM, Wuskell JP (1996) Dye screening and signal-to-noise ratio for retrogradely transported voltage-sensitive dyes. J Neurosci Methods 70:121-129.

Vassar R, Ngai J, Axel R (1993) Spatial segregation of odorant receptor expression in the mammalian olfactory epithelium. Cell 74:309-318.

Vassar R, Chao SK, Sitcheran R, Nuñez JM, Vosshall LB, Axel R (1994) Topographic organization of sensory projections to the olfactory bulb. Cell 79:981-991.

Yokoi M, Mori K, Nakanishi S (1995) Refinement of odor molecule tuning by dendrodendritic synaptic inhibition in the olfactory bulb. Proc Natl Acad Sci USA 92:3371-3375.

Youngentob SL, Kent PF, Sheehe PR, Schwob JE, Tzoumaka E (1995) Mucosal inherent activity patterns in the rat: evidence from voltagesensitive dyes. J Neurophysiol 73:150-160.

Zhao H, Ivic L, Otaki JM, Hashimoto M, Mikoshiba K, Firestein S (1998) Functional expression of a mammalian odorant receptor. Science 279:237-242. 